\title{
粗Sociología
}

Follegati Montenegro, L. (2020). ¿Qué democracia? Feminismo y política en el Chile de los 80s. Revista de Sociología, 35(1), 56-68. doi: 10.5354/0719529X.2020.58107

\section{¿Qué democracia? Feminismo y política en el Chile de los 80s}

\author{
[What democracy? Feminism and politics in the Chile of the 80s] \\ Luna Follegati Montenegro(i) \\ Pontificia Universidad Católica de Chile
}

\begin{abstract}
Resumen
En los últimos años el movimiento feminista se articuló como un actor importante que ha cuestionado aspectos sustantivos del régimen político y económico en Chile. La pregunta por la democracia desde el feminismo vuelve a tener un lugar especial, particularmente en relación a las formas en que los feminismos replantean el cauce normal de la política, las esferas de acción y la índole de las transformaciones. En ese sentido, adquieren vigencia las propuestas teórico-políticas del feminismo en Chile de los 80, las cuales propusieron una alternativa de democratización desde el feminismo. Este trabajo analiza estas propuestas, particularmente sobre conceptos como lo político y democrático en autoras asociadas al pensamiento socialista como Julieta Kirkwood, Adriana Muñoz, y Natacha Molina. A partir de este ejercicio analítico se muestra la conformación de un pensamiento teórico político feminista que desarrolló una visión propia sobre la política, la democracia y la democratización a partir de la reflexión en los espacios académicos y de la práctica y experiencia político-social.
\end{abstract}

Palabras clave: movimiento feminista, dictadura, democratización, feminismo, género.

\begin{abstract}
In recent years, the Chilean feminist movement has played an important role in challenging major aspects of national political and economic governance. The question of democracy, approached from a feminist perspective, has once again achieved prominence, particularly in terms of how feminism rethinks the normal course of politics, its spheres of operation, and the nature of its transformations. The theoretical and political proposals of feminism in 1980s Chile, which advocated an alternative form of democratization, have once again become valid. This article analyzes those proposals, focusing on concepts of politics and democracy as expressed by socialist Chilean authors such as Julieta Kirkwood, Adriana Muñoz, and Natacha Molina, revealing the structure of a feminist political theory that has developed its own vision of politics, democracy, and democratization based on academic reflection and sociopolitical practice and experience.
\end{abstract}

Keywords: feminist movement, dictatorship, democratization, feminism, gender.

Contacto: La comunicación sobre este artículo debe ser enviada a Luna Follegati Montenegro, email lufollegati@uc.cl

Financiamiento: Agencia Nacional de Investigación y Desarrollo (ANID), PIA, Proyecto Anillos en Ciencias Sociales y Humanidades, The Production of the Gender Norm, SOC 180023. 


\section{INTRODUCCIÓN}

Durante los últimos años el escenario de la política chilena ha estado particularmente convulso. Movimientos sociales y reivindicaciones han cuestionado diversos aspectos del régimen político y económico heredados del período de la dictadura militar. Uno de los ámbitos que más ha sido flanco de críticas es la calidad y legitimidad de la democracia, cuestionamientos que quedaron expuestos en las reivindicaciones y consignas de la revuelta chilena de octubre 2019. Analizar las bases que erigieron la democracia chilena actual se vuelve un imperativo, toda vez que los nuevos movimientos sociales de la última década han exigido una reformulación del sistema democrático en su conjunto, apelando a las formas, límites y mecanismos desde donde se comprende $\mathrm{y}$ desarrolla la política. Los feminismos no han estado exentos de esa crítica y reflexión, desde la irrupción del movimiento feminista estudiantil del 2018, como también en las multitudinarias marchas conmemorativas del 8 de marzo, demostrado la capacidad de organización y la fuerza de las demandas del movimiento, cuyas exigencias radican en transformaciones en el plano político, económico y social desde la perspectiva feminista.

A partir de lo anterior han proliferado propuestas teóricas y académicas que han abordado la relación entre la democracia y la nueva constitución (Brito, 2020), el estallido social (Castillo, 2019), reflexiones sobre el movimiento feminista estudiantil (Zerán, 2018), o sobre política y mujeres (Arce, 2018). En todos ellos, la referencia tanto a la historia del movimiento en Chile, como a las teóricas chilenas ha sido clave. Particularmente atingente son los trabajos de la feminista chilena Julieta Kirkwood que, publicados y reeditados en la actualidad (2010, 2017, 2019), han ayudado a destacar el carácter histórico-político del movimiento. En este contexto, este trabajo busca problematizar la relación entre política y democracia desde la perspectiva de algunas feministas socialistas esgrimidas en los años 80. Este vínculo es relevante pues el movimiento feminista chileno ha sido un actor sustantivo en la activación y crítica político social de los últimos años, así como en el período dictatorial (Kirkwood, 2010; Largo, 2014).

Las propuestas teórico-políticas del feminismo en Chile -coetáneas a las discusiones sobre la transición a la democracia-apelaron igualmente a una transformación sustantiva del régimen político, proponiendo a la vez una alternativa democrática desde el feminismo. A 30 años del retorno democrático, los feminismos contemporáneos revisitan la producción teórica de la tradición de los 80 desarrollando nuevos requerimientos y exigencias hacia el sistema democrático, esta vez, con una fuerte crítica al neoliberalismo. Durante este ejercicio analítico se mostrará cómo los feminismos han cuestionado permanentemente un orden democrático, constituyéndose como un corpus teórico mediante una crítica social y reflexión intelectual que cuestiona las estructuras del sistema político. Estos aspectos señalan la 
continuidad de la crítica feminista desde los 80 como una forma de comprender la política, apelando a un orden político social distinto al actual. Así, en primer lugar, se plantearán algunos aspectos relevantes del pensamiento feminista en los 80, interrogados desde preguntas y problemas actuales. En un segundo momento se profundizará en el cruce entre política y feminismo, abordando definiciones y propuestas analíticas. Y finalmente, se abordará el problema de la democracia y feminismo, para esgrimir conclusiones sobre las perspectivas analíticas actuales que desprendemos desde el pensamiento feminista de los 80 .

\section{REVISITANDO LOS 80: PREGUNTAS ACTUALES DESDE LA EXPERIENCIA ANTERIOR}

Luego del estallido social chileno de octubre, Brito (2020) menciona un nudo que parece pertinente posicionar como apertura: "Relevando la herida dictatorial nos volvemos a preguntar las dudas feministas de los años ochenta: ¿será un partido apto para representar nuestras 'necesidades' como feministas? ¿se constituirá un espacio político donde tengamos efectivamente representatividad y expresión los movimientos sociales?" (p. 14). Para Brito, el movimiento feminista se reconoce por el disenso, que presupone defender la construcción de la democracia por y para quienes han vivido históricamente situaciones de explotación y opresión. En Brito (2020) se ensaya sobre el cruce entre constitución y feminismo, entrecruzando las letras de Carmen Berenguer (poeta, cronista y artista visual chilena) sobre la dictadura con las palabras de las voceras de la coordinadora 8M; apelando a la forma en que el movimiento feminista ha exigido condiciones de posibilidad para procesos democráticos sustantivos, componiendo como referencia al propio movimiento feminista de los 80 y agrupaciones como Mujeres por la Vida.
Las sincronías aparecen en la continuidad de un orden represivo, las resistencias a dicho orden y la lucha contra la impunidad. En este sentido, preguntas sobre el autoritarismo y patriarcado parecen tener un especial eco. La experiencia feminista anterior tiene un significado sustantivo para la comprensión política del presente. Más que un ejercicio histórico formal, se apela a una memoria política feminista. Por esta razón es preciso volver sobre los pasos de las feministas de los 80 que advierten sobre experiencias pertinentes para el momento actual: qué democracia, qué política y cómo el feminismo se entrecruza entre ambos.

El feminismo en Chile, durante los 80 en tanto teoría y acción, fue parte de la discusión alertando sobre el problema autoritario y la necesidad de la democracia desde una política feminista, política en tanto irrupción de un orden establecido. Castillo (2014) ha señalado la potencia de las mujeres en la escena pública en tanto gesto político de la toma de palabra, advirtiendo que "sólo hay política cuando una 'parte no parte', excluida/invisible, politiza su 
situación y perturba la estructura establecida del orden de representación político/policial, afirmándose como representante universal y exigiendo la rearticulación de su posición particular" (p. 19). Desde este lugar, el gesto político feminista se constituye al figurar esa posibilidad de toma de palabra, cuestión que Castillo reconoce a comienzos de siglo XX, pero que también podemos identificar en la conformación del movimiento en los 80 con la irrupción de un lenguaje feminista que se encarna en la forma de movimiento y acción. Castillo (2016) refuerza esta sintonía entre política y emancipación, en tanto asalto feminista que desde Kirkwood "adquiere las formas de la rebeldía, la protesta y la búsqueda de los medios necesarios para hacer de la política una lengua que hable también de la opresión y la violencia patriarcal" (p. 10).Con ello, es posible inferir que el ingreso de las mujeres a la política se fragua en esa disputa por la distribución y reasignación de lugares y formas de distribución de identidades, de espacios, tiempos y lenguas, pero que interroga a las mujeres y a la política, estructurándose el feminismo como un discurso político de subversión en el contexto socio simbólico de represión autoritaria (Castillo, 2007), pero también bajo la idea de subvertir un orden establecido. Los 80 se identifican como una década donde la temática de la democracia se problematiza en todas sus dimensiones. Para las feministas latinoamericanas, las dictaduras del cono sur obligaron al cruce problemático entre derechos y democracia desde las mujeres, cuestión que se vio reflejada en los Encuentros Feministas Latinoamericanos y del Caribe, desde el año 1981 en adelante (ver, Toro, 2009). Las propuest as feministas latinoamericanas tomaron un ribete distinto a la tradicional segunda ola del norte, cuestión dada por la coyuntura histórica que los aquejaba. En el caso chileno la problematización sobre la democracia será fundamental en las producciones de Kirkwood (1986, 1987, 2017), pero también tendrá un lugar en las propuestas de Muñoz (1987) y Molina (1986) quienes, afiliadas a un pensamiento y militancia socialista, establecen reflexiones sobre la relación entre feminismo y democracia. Además, se suman a otras elaboraciones de sectores del movimiento, como el Pliego de las Mujeres presentado a la Asamblea de la Civilidad en 1986 (Gaviola, Largo, \& Palestro, 1994), las propuestas de la Concertación Nacional de las Mujeres a la Democracia, o el libro editado por Montecino y Rossetti (1990). Así, las producciones feministas fueron un ámbito más de producción teórico-política sobre el devenir de la democracia en Chile.

\section{FEMINISMO Y POLÍTICA}

A finales de los 70, el cruce entre democracia y socialismo atraviesa un momento político intelectual particular. Como es sabido, se instala la noción de un nuevo socialismo democrático de acuerdo a los planteamientos de la renovación socialista, inaugurando la década de los 80 un escenario 
rediseño político y teórico (Follegati, 2018b). Para Puryear (2016), durante esos años el pensamiento y discurso político chileno se fue intelectualizando, lo que en muchos casos coincidió con la labor de renovación política (Arrate, 1985; Flisfisch, 1984; Moulian, 1983; Tironi, 1984). En ese contexto, la distinción entre democracia y democratización fue un eje de la discusión de la época, en exponentes como Garretón (1988) o Lechner (1988), apelando a la diferencia entre una institucionalidad política democrática, y la forma en que se profundiza la democracia en las relaciones sociales y estructuras institucionales alteradas por el autoritarismo.

Aun cuando algunas de las exponentes feministas fueron parte del proceso de renovación, la reflexión feminista se mantuvo en un carril y ámbito diferenciado, apuntado a temas similares, pero abordándolo desde otras aristas. Por una parte, el problema de la democratización estuvo vinculado a la intención de transformación propiciada por el movimiento feminista en Chile, por ejemplo, a través del Manifiesto Feminista de 1983 cuyo primer párrafo es categórico: "Ningún proyecto de democratización será viable, sólido ni justo, si no enfrenta los problemas de discriminación que sufrimos el 50 por ciento de la población por el hecho de ser mujeres. Esto no significa negar los efectos de la desigualdad social" (Gaviola et al., 1994, p. 234). La incorporación de las mujeres a la disputa política implicó un pensamiento y propuesta sobre la democracia. En los diversos textos de la época, manifiestos o pliegos de mujeres, se percibe la vinculación entre la participación política de las mujeres con su compromiso democrático, elemento que se percibe y manifiesta a través de un cuestionamiento profundo a la matriz autoritaria presente en la sociedad. A la vez, plantean la condición política de aspectos relevados a un segundo plano, como la vida cotidiana-privada (Gaviola et al., 1994).

El pensamiento feminista, elaborado al calor del movimiento, explicita su filiación política en tanto exige una transformación de la estructura social en su conjunto, siendo este un rasgo político per se. Para Kirkwood (1987) lo político del feminismo implica una apropiación, como primer gesto de resignificación de las formas tradicionales:

La política misma, tan extraña, tan ajena, tan cosa de hombres para la gran masa de las mujeres, adquiere nuevas dimensiones $\mathrm{y}$ perspectivas inexploradas hasta ahora. La política, asumida por las mujeres, significa el derecho a opinar, cuestionar, proponer y participar en la abolición de la sociedad presente y en la construcción de la sociedad futura. Y significa también, el derecho irrenunciable a destruir su propia discriminación y explotación y a reconstruir su condición futura (p. 66).

La política como propuesta y necesidad articulada por la demanda de derechos, pero que también apuesta por la transformación implica "repensar la organización de la vida cotidiana de mujeres y de hombres" (Kirkwood, 1987, p. 86). Así, el ejercicio político sería una forma de democratización desde el feminismo, un reordenamiento radical a las estructuras políticas y sociales, que se visibiliza a 
través del problema de la exclusión. Muñoz (1987) recalca el rol del movimiento en tanto fuerza política que logra articular una concepción alternativa de sociedad, que implique transformaciones a nivel estructural pero también desde lo individual, sus derechos, necesidades y vida cotidiana. Para Muñoz (1987), lo novedoso de los nuevos movimientos sociales de la época estuvo dado en la forma en que se aproximan a la política desde una comprensión del poder que lo reconoce en todos los ámbitos y dimensiones de la sociedad. La política implica así una transformación de las relaciones de poder, y por lo mismo "empieza a ser concebida como expresión y como práctica de todos aquellos sectores oprimidos y subordinados en todo ámbito y dimensión en que se expresa el poder" (Muñoz, 1987, p. 74). La misma descripción contiene un fuerte énfasis en la práctica de los sujetos, en tanto gestores de un proyecto de cambio. La política así vista, se vincula estrechamente con una dimensión social, descentrando la focalización de esta en el Estado y partidos políticos, revalorando las expresiones políticas que emergen desde lo social:

La política se enriquece y complejiza porque los espacios propios del mundo social, como las relaciones personales, la sexualidad, lo doméstico, la violencia, vale decir todo aquello que constituye lo privado y la cotidianeidad, empieza a estar presente y a impregnar la práctica política. En este sentido se humaniza lo público y se politiza lo privado (Muñoz, 1987, p. 74).

La conceptualización de una política desde lo privado se construye en sintonía con la resignificación de los roles y tareas asignadas a lo femenino, en estrecha relación con la politización de lo cotidiano, territorial y autónomo desde el pensamiento feminista. Pensar la política desde el movimiento social de mujeres implicó ensayar una contrapropuesta de sociedad, o al menos, plantear un cuestionamiento profundo de las bases de las formas tradicionales (partidos y Estado), que también condujo a un profundo ejercicio de teorización en torno a los contornos de lo político y democrático desde el feminismo. En este sentido, es posible señalar que la reflexión feminista combinó una tríada: la pregunta por lo político de la acción feminista, la cuestión de la democracia para las mujeres y las formas de democratización social.

\section{FEMINISMO Y DEMOCRACIA}

Junto con la activación feminismo en tanto movimiento, se restituye también la pregunta por la democracia para las mujeres (Kirkwood, 1987). Interrogación que contenía una respuesta marcada por la rebeldía, al constatar la diferencia entre lo que se planteaba como igualdad para el género humano y lo que efectivamente vivencian las mujeres. Feminismo, democracia y política presentan un nudo cuya condición de exterioridad y develamiento ocurre en medio del período autoritario, y a costa de éste, pero constituye además una reflexión que surge desde los espacios de 
reflexión, organización y reunión de mujeres a partir de los últimos años de la década del 70 .

El problema político feminista deriva también en una crítica sobre la forma del ordenamiento político en el país, a través de una organización de la vida cotidiana de hombres y mujeres mediante la articulación de ámbitos de acción excluyentes y rígidos: lo público/político y lo privado/doméstico. Este aspecto también es abordado por Sánchez (2005) al señalar la particularidad del feminismo en dictadura, ya que "el significado de hacer política desde la condición de género de las mujeres sobre la base de sus carencias (...) permite integrar dos formas de experiencias antes escindidas: la de lo público y la de lo privado" (pp. 71-72). Molina (1986) reflexiona cómo el golpe militar en Chile afecta lo cotidiano, convirtiéndolo en el espacio político para toda la sociedad, cuestión que posibilita la integración de estos problemas en la nueva propuesta democrática feminista. Paradojalmente, el feminismo se recompone en la dictadura chilena en tanto "el autoritarismo ofrece una oportunidad para redefinir la democracia que se quiere construir, desterrando de ella toda forma de subordinación” (Molina, 1986, p. 15). Al igual que Kirkwood, enfatiza en la necesidad de descomponer la dicotomía público-privada como respuesta a la pregunta sobre mujeres y políticas, pues "de ahí surgen propuestas que se caracterizan más por un cuestionamiento de los proyectos de cambio que se ofrecen como alternativa, que por un modelo de legalidad democrática" (Molina, 1986, p. 39) como el de la transición.

La democracia desde el movimiento de mujeres está asociada a dinámicas territoriales o sectoriales, pues desde allí se propone una nueva identidad que busca cambiar la relación entre mujer y política. Las organizaciones territoriales propician una práctica femenina que apuesta por configurar una alternativa a la institucionalidad democrática. En esto, la acción de las mujeres "manifiesta una ruptura con la centralidad política como acción privativa de las instancias estatales y, de alguna manera, promueve cambios en la lógica del sistema de partidos que, en cierto modo, reproduce dicha centralidad" (Molina, 1986, p. 41). El movimiento feminista en Chile, en su acepción territorial, comprende la posibilidad de cuestionar la normalidad política democrática asignando un componente de democratización vinculado al espacio privado, alterando así los regímenes de producción y reproducción de las relaciones sociales: la política feminista visibiliza cuestiones como la familia, sexualidad y cotidianeidad desde la cual se esgrimen propuestas de cambio político para amplios sectores sociales.

Una noción similar es abordada por Muñoz (1987), donde la forma democrática debería combinar la participación directa e indirecta como clave para iniciar un proceso de democratización del Estado. Prefigurando ya un futuro programa de gobierno, establecía una continuidad entre el rol de las mujeres de sectores populares en las formas de organización social territorial y su futuro protagonismo en la ejecución y gestión de políticas públicas desde el Estado, pero en el ámbito comunal. El énfasis correspondería a la vinculación entre democracia y prácticas participativas, cuestión que lograría incorporar a las mujeres en una formulación real 
de la democracia, a través de un equilibro entre partido y organizaciones sociales (Muñoz, 1987). En la práctica, el énfasis democrático se vinculaba más a una democratización del Estado desde la socialización de políticas públicas y la gestión de éstas, incorporando a las mujeres a la acción del Estado, lo cual rompería la dominación patriarcal histórica e institucional sobre la mujer. El movimiento feminista chileno, desde la perspectiva de Muñoz, pasaría así a un momento de involucramiento conformándose como parte orgánica del proceso global de decisiones del país, más que como grupo de presión, cuestión que contenía en si una propuesta política cuya apuesta era el diseño, planificación y negociación de políticas orientadas a eliminar la subordinación de género y clase. Ciertamente, dicha visión -más cercana a lo que finalmente se traducirán algunas de las propuestas de las mujeres de la Concertacióncorrespondió a una reflexión que integró formas directas y representativas de democracia: "lograr que estas dejen de ser dos formas separadas de expresión de la democracia, para llegar a constituir dos elementos dialécticamente interrelacionados de una sola autoridad democrática: la democracia participativa" (Muñoz, 1987, p. 57).

Aspectos como conciliación e integración de formas directas y representativas aparecen como conceptos cada vez más propicios para referirse a la propuesta democrática desde teóricas socialistas que abrazaban el feminismo. Cruce interesante, ya que en Muñoz (1987) o Molina (1986) es posible percibir un doble lenguaje: aquel que cuestiona e interpela a las estructuras político-patriarcales, como también uno que dialoga sobre las posibles formas de gobierno basadas en la representación de las mujeres, y con ello, las formas de institucionalización de las demandas. Estos trabajos muestran la complejidad de un período que buscó conectar una experiencia política feminista con los causes de la política que se avecinada, cuestión que muestra la compleja, tensa y conflictiva conexión que se generó entre el feminismo en vías de institucionalización y la normalización democrática de la transición de los 90 . Un ejemplo de esto es que los movimientos sociales y el feminismo en particular, a diferencia de los partidos, no se propone cambiar la sociedad a través de la destrucción del Estado y la toma del poder, sino que a través de la búsqueda de legitimidad y consenso en la sociedad civil en torno a una propuesta alternativa de sociedad gestada por ella misma (Muñoz, 1987, p. 20).

La legitimidad del movimiento feminista en Chile se presenta como un aspecto constitutivo y clave para comprender a las mujeres en tanto grupo que presiona o exige a la democracia; esta vez, vinculada a la concreción de una fuerza política gestora y constructora del proyecto democrático naciente en Chile. Ciertamente, esta visión ya contenía una compresión determinada sobre la acción de las mujeres que establecía un lugar y por tanto, una circunscripción al accionar de las mujeres, ya distanciada del tono más subversivo de Kirkwood. En palabras de Muñoz, "mantener las organizaciones actuales de mujeres y la movilización social en tiempos de democracia es uno de los puntos más oscuros y, a la vez, el gran 
fantasma que surge de las experiencias anteriores" (1987, p. 39). El feminismo, en tanto irrupción, rebeldía y lucha, tendrá que mantenerse en suspenso frente al discurso consensual de los 90, que termina por constituirse como hegemónico.

Así, la transición a la democracia inaugura un nuevo período que se caracteriza como un complejo episodio para el movimiento feminista chileno. La llegada de los feminismos en la institucionalidad estatal produjo no sólo un silencio crítico, sino que también un desmembramiento en tanto movimiento aquejado por los diversos caminos estratégicos del feminismo que se aventuraron a comienzos de los 90 (Richard, 2001; Ríos, Godoy, \& Guerrero, 2003). Como señala Forstenzer (2013), algunas feministas deciden darle prioridad a la ocupación del Estado en la política formal o la cooperación desde la sociedad civil, mientras que otras consideran preciso constituirse en fuerza de presión autónoma. Podría aseverarse, que la desarticulación del movimiento feminista chileno fue parte del proceso de recomposición de la democracia en Chile, y que ocurrió de forma similar a las otras experiencias de articulación social surgidas en dictadura. El retorno democrático en los 90 implicó una política que buscaba resolver las demandas propugnadas en la década anterior en el contexto antidictatorial mediante el Estado y sus políticas públicas, vehiculizando las acciones a través del Servicio Nacional de la Mujer (SERNAM) creado en 1991 para resolver tales temáticas. En este sentido, Schild (2016) señala que en la década de los 90 se consolidó un "feminismo de lo posible", que enlazó una política de mujeres liberal y pragmática, con una agenda en general de democratización cautelosa. Así, las políticas de género no sólo desactivaron el énfasis contestatario y subversivo del feminismo de los 80 en los sectores populares, sino que tecnificaron su potencia mediante una política gubernamentalizada del orden (Follegati, 2018a; Schild, 2016). A través de principios neoliberales para el reconocimiento de la autonomía de las mujeres, se prefirió una producción más técnico instrumental que crítica (Valdés, 1993) y con ello las propuestas rupturistas del feminismo ingresaron en un complejo momento de diversificación frente al orden transicional.

\section{CONCLUSIONES}

A treinta años del retorno democrático los feminismos actuales revisitan la producción teórica de la tradición de los 80 , desarrollando nuevos requerimientos y exigencias hacia el sistema democrático, con un énfasis antineoliberal. En este sentido, los feminismos contemporáneos en Chile han sostenido una crítica social y reflexión intelectual que interpela las estructuras del sistema político, cuestión que señala que la continuidad del feminismo también se explicita en tanto emergen problemas políticos teóricos que apelan temas similares, como la cuestión de la democracia o el cuestionamiento a la dicotomía público/privada. Los nudos de Kirkwood (1982, 
1983, 1986, 1987) parecen reiterarse, pero también vuelven con inusitada vigencia en tanto corresponden a preguntas centrales de toda teoría feminista. Estos aspectos evidencian que en la actualidad asistimos a un momento clave y sustantivo para el movimiento: advertimos la potencialidad de una política feminista en tanto cuestionamiento y alternativa a un orden establecido que produce pensamiento teórico en tanto que es movimiento activo.

El pensamiento feminista en los 80 muestra la estrecha relación entre feminismo y democracia, a partir de lo cual es plausible sostener que la democracia posee un efecto sobre el feminismo y viceversa. No es casual la vinculación entre la contingencia histórica autoritaria y la activación del movimiento, como tampoco el ejercicio de entrada y salida del feminismo en la escena pública, pues la propia definición política del movimiento revelaría el carácter de su discurso en tanto rebeldía: cuestiona la figura de lo permitido y lo intruso, como también el lugar de exclusión y subordinación a partir de lo sexual. Así, la relación entre feminismo y democracia podría comprenderse desde el lugar que ocupa el problema de la exclusión, o bien, como se considera la relación entre igualdad y democracia. Si nos preguntamos por la inclusión de mujeres en las esferas tradicionales de lo político, bastaría una democracia tradicional que fomentara el ingreso de las mujeres, en tanto grupo excluido. En cambio, si por democracia se comprende también una forma de organización social que permea las estructuras sociales, debemos ensayar una definición que posibilite formas de democratización de las esferas sociales en su conjunto. Así, democracia comprendida desde lo privado y cotidiano en conjunto y a la vez: "democracia en el país y en la casa" como consagra el lienzo del movimiento feminista de 1983.

Perspectivas como estas estuvieron en tensión a finales de los 80. La forma que adquiere el pensamiento y discurso feminista también habla de la caracterización del movimiento, la vigencia política de éste y la radicalidad de la transformación que completa su forma. Relevar las cartografías del pensamiento de los 80 constituyen hoy una necesidad tanto para el movimiento feminista chileno del presente, como también para el ejercicio de memoria histórica que busca comprender el pensamiento sociopolítico feminista chileno. Como señala Kirkwood (1987), el saber feminista es un bricolaje, que compone diferentes lenguas, contextos y saberes. En este sentido, el pensamiento crítico feminista que emerge durante la dictadura militar en Chile posibilitó el cuestionamiento a las estructuras de representación tradicionales, como también la politización de problemas (e.g., violencia doméstica) y espacios (público/privado), contextualizados a través de la pregunta por la dimensión política del cuerpo. Desde esta perspectiva, los feminismos propusieron un escenario de cambio que subvierte el componente normativo de la democracia y política en tanto procedimiento, cuestión que convierte al feminismo como un pensamiento teórico político que juega con el desbarajuste de las formas tradicionales. Por ello, su constante marginación como pensamiento no permitido bajo el canon de lo 
normal. El feminismo se fragua en esa figura del borde, y se articula políticamente como derecho irrenunciable: es político, parafraseando a Kirkwood, en tanto que busca abolir un orden presente. Es político también, en tanto que considera una práctica social feminista, una desde las organizaciones que proliferaron desde lo social apuntando a otra forma de democratización. Así, los discursos de finales de los 80 presentarán una tensión, entre aquellos feminismos que continúan con discursos y prácticas que apuestan por una transformación radical de las estructuras patriarcales, y otros que comienzan a ensayar nuevas formas de incorporación de las mujeres y el movimiento en los cánones de la política democrática que se avecina. Ciertamente la democracia de los 90 no respondió a la radicalidad subversiva del feminismo de los 80 , tampoco de conceptualizaciones que apostaban por una democratización mayor de aquella que finalmente se fraguó. Sin embargo, hoy retomamos sus reflexiones en un contexto donde la crisis de legitimidad democrática requiere del movimiento feminista en Chile una acción sustantiva y un pensamiento propio. En esto, es preciso revisitar la experiencia previa y reconstruir desde allí la continuidad de un problema: la relación entre feminismo y democracia. La actualidad nos llama a continuar una reflexión sobre la democracia que contenga el problema de la democratización en su interior. La democracia sin resignificación de la estructura social no es sinónimo de igualdad para las mujeres, como tampoco lo es desvincularla de las marcas de clase, raza o nacionalidad. El pensamiento feminista debe continuar la pregunta por la politización de lo privado cuya clave de respuesta ocurre desde el movimiento: la experiencia, la práctica y la asociatividad constituyen hoy, como ayer, las herramientas predilectas para la teorización feminista.

\section{REFERENCIAS}

Arce, J. (Ed). (2018). El Estado y las mujeres. El complejo camino hacia una necesaria transformación de las instituciones. Santiago, Chile: ADN Editores.

Arrate, J. (1985). La fuerza democrática de la idea socialista. Santiago, Chile - Barcelona, España: Ediciones Documentas, Ediciones del Ornitorrinco.

Brito, S. (Ed.). (2020). Por una constitución feminista. Santiago, Chile: Editorial Pez Espiral.
Castillo, A. (2007). Julieta Kirkwood. Políticas del nombre propio. Santiago, Chile: Editorial Palinodia.

Castillo, A. (2014). El desorden de la democracia.

Partidos políticos de mujeres en Chile. Santiago, Chile: Editorial Palinodia.

Castillo, A. (2016). Disensos feministas. Santiago, Chile: Editorial Palinodia.

Castillo, A. (2019). Asamblea de los cuerpos.

Santiago, Chile: Sangría Editorial. 
Flisfisch, A. (1984). Hacia un realismo político distinto. (Documento de Trabajo $\mathrm{N}^{\mathrm{o}} 219$ ). Santiago, Chile: FLACSO.

Follegati, L. (2018a). El constante aparecer del movimiento feminista. Reflexiones desde la contingencia. En F. Zerán (Ed.), Mayo feminista. La rebelión contra el patriarcado (pp. 77-90). Santiago, Chile: LOM Ediciones.

Follegati, L. (2018b). La transición a la democracia en Chile: Genealogía de un concepto (19731990). (Tesis doctoral, Universidad de Chile, Santiago, Chile). Recuperado de http://repositorio.uchile.cl/handle/2250/171031

Forstenzer, N. (2013, septiembre). Ser feminista en el Chile actual: Ambigüedades y dilemas de las reivindicaciones de igualdad de género. Ponencia presentada en coloquio Chile actual. Gobernar y resistir en una sociedad neoliberal. Del caso Pinochet al gobierno de Sebastián Piñera (1998-2013). Grenoble, Francia.

Garretón, M. A. (1988). El plebiscito de 1988 y la transición a la democracia. Santiago, Chile: FLACSO.

Gaviola, E., Largo, E., \& Palestro, S. (1994). Una historia necesaria. Mujeres en Chile 19731990. Santiago, Chile: ASDI.

Kirkwood, J. (1982). Feminismo y participación política. (Documento de trabajo $\mathrm{N}^{\circ} 159$ ). Santiago, Chile: FLACSO.

Kirkwood, J. (1983). El feminismo como negación del autoritarismo. (Material de discusión $\mathrm{N}^{\circ}$ 52) Santiago, Chile: FLACSO.

Kirkwood, J. (1986). Ser política en Chile. Los nudos de la sabiduría feminista. Santiago, Chile: Editorial Cuarto Propio.
Kirkwood, J. (1987). Tejiendo rebeldías. Escritos feministas. Santiago, Chile: Centro de Estudios de la Mujer, La Morada.

Kirkwood, J. (2010). Ser politica en Chile. Las feministas y los partidos. Santiago, Chile: LOM.

Kirkwood, J. (2017). Feminarios. Viña del Mar: Asociación communes.

Largo, E. (2014). Calles caminadas. Anverso y reverso. Santiago, Chile: DIBAM, Centro de Investigación Diego Barros Arana.

Lechner, N. (1988). Los patios interiores de la democracia. Subjetividad y política. Santiago, Chile: FLACSO.

Montecino, S., \& Rossetti, J. (Eds.). (1990). Tramas para un nuevo destino: propuestas de la Concertación de Mujeres por la Democracia. Santiago, Chile: Concertación de Mujeres por la Democracia.

Molina, N. (1986). Lo femenino y lo democrático en el Chile de hoy. Santiago, Chile: Centro de Estudios Económicos y Sociales VECTOR.

Moulian, T. (1983). Democracia y socialismo en Chile. Santiago, Chile: FLACSO.

Muñoz, A. (1987). Fuerza feminista y democracia. Utopía a realizar. Santiago, Chile: Ediciones Documentas.

Puryear, J. M. (2016) Pensando la política: Intelectuales y democracia en Chile, 19731988. Santiago, Chile: Uqbar Editores.

Richard, N. (2001). La problemática del feminismo en los años de la transición en Chile. En D. Mato (Ed), Estudios latinoamericanos sobre cultura $y$ transformaciones sociales en tiempos de globalización (pp. 227-239). Buenos Aires, Argentina: CLACSO. 
Follegati Montenegro, L. (2020). ¿Qué democracia? Feminismo y política en el Chile de los 80s. Revista de Sociología, 35(1), 56-68. doi: 10.5354/0719529X.2020.58107

Ríos, M., Godoy, L., \& Guerrero, E. (2003). ¿Un nuevo silencio feminista? La transformación de un movimiento social en el Chile postdictadura. Santiago, Chile: Editorial Cuarto Propio.

Sánchez, C. (2005). Escenas del cuerpo escindido. Ensayos cruzados de filosofía, literatura $y$ arte. Santiago, Chile: Editorial Cuarto Propio, Centro de Estudios de la Mujer.

Schild, V. (2016). Feminismo y neoliberalismo en América Latina. New Left Review, 96(1-2), 63-99. Recuperado de http://newleftreview.es/issues/96/articles/ve ronica-schild-feminismo-y-neoliberalismoen-america-latina.pdf

Tironi, E. (1984). La torre de Babel: Ensayos de crítica y renovación política. Santiago, Chile: Ediciones Sur.
Toro, M. S. (2009). Debates feministas latinoamericanos: institucionalización, autonomía. Santiago, Chile: Libros La Calabaza del Diablo.

Valdés, T. (1993). El movimiento social de mujeres y la producción de conocimientos sobre la condición de la mujer. (Documento de trabajo $\left.{ }^{\circ} 43\right)$. Santiago, Chile: FLACSO.

Zerán, F. (Ed.). (2018). Mayo feminista. La rebelión contra el patriarcado. Santiago, Chile: LOM Ediciones.
Manuscrito recibido: 02-03-2020

Manuscrito aceptado: 10-06-2020 